\title{
Managing atrial fibrillation in the elderly: critical appraisal of dronedarone
}

\author{
This article was published in the following Dove Press journal: \\ Clinical Interventions in Aging \\ 29 December 2011 \\ Number of times this article has been viewed
}

\section{Paula Trigo \\ Gregory W Fischer}

Department of Anesthesiology, Mount Sinai School of Medicine, New York, NY, USA
Correspondence: Gregory W Fischer Division of Cardiothoracic Anesthesiology, Mount Sinai School of Medicine, KCC 8th Floor, One Gustave L Levy Place, Box 1010, New York, NY, USA Tel + I 2 I $224 \mid 7473$

Fax + I 2124262009

Email gregory.fischer@mountsinai.org

\begin{abstract}
Atrial fibrillation is the most commonly seen arrhythmia in the geriatric population and is associated with increased cardiovascular morbidity and mortality. Treatment of the elderly with atrial fibrillation remains challenging for physicians, because this unique subpopulation is characterized by multiple comorbidities requiring chronic use of numerous medications, which can potentially lead to severe drug interactions. Furthermore, age-related changes in the cardiovascular system as well as other physiological changes result in altered drug pharmacokinetics. Dronedarone is a new drug recently approved for the treatment of arrhythmias, such as atrial fibrillation and/or atrial flutter. Dronedarone is a benzofuran amiodarone analog which lacks the iodine moiety and contains a methane sulfonyl group that decreases its lipophilicity. These differences in chemical structure are responsible for making dronedarone less toxic than amiodarone which, in turn, results in fewer side effects. Adverse events for dronedarone include gastrointestinal side effects and rash. No dosage adjustments are required for patients with renal impairment. However, the use of dronedarone is contraindicated in the presence of severe hepatic dysfunction.
\end{abstract}

Keywords: atrial fibrillation, elderly, antiarrhythmic agents, amiodarone, dronedarone

\section{Introduction}

Atrial fibrillation is the most common arrhythmia requiring medical therapy, and increases in prevalence with advancing age. ${ }^{1}$ The long-term incidence of hospitalization related to atrial fibrillation is high, which makes prevention a key factor. ${ }^{2}$ According to a study conducted by Go et al, atrial fibrillation is more common in men than in women, with an increase in prevalence in persons aged 80 years or older. They projected that this prevalence will increase to more than 5.6 million by the year 2050 , with more than $50 \%$ of affected individuals being aged 80 years or older. ${ }^{3}$ The presence of atrial fibrillation translates into significant morbidity and mortality for the patient. Stroke, thromboembolism, heart failure, impaired quality of life, and recurrent hospitalizations are frequently encountered complications in patients with atrial fibrillation. ${ }^{4}$ Of all these adverse events, stroke is by far the most feared, often leading to severe disability or even death. ${ }^{5}$ The Framingham study showed atrial fibrillation is an independent risk factor for stroke. ${ }^{6}$ It is estimated that atrial fibrillation is responsible for over 60,000 strokes each year in the United States. ${ }^{7}$ Stroke is the second most common cause of death in the world. Almost $90 \%$ of deaths caused by stroke occur in people over 65 years of age. ${ }^{8}$ Consequently, contemporary therapy for atrial fibrillation has two major goals, ie, symptom relief and prevention of complications, including stroke and heart failure. 
Treatment of patients with atrial fibrillation poses a great challenge and can broadly be divided into two major therapeutic strategies, ie, rate control and rhythm control. Interestingly, two of the largest studies to date focusing on this topic, ie, the AFFIRM (Atrial Fibrillation Follow-up Investigation of Rhythm Management) and AF-CHF (Atrial Fibrillation in Congestive Heart Failure) trials, were both unable to show that antiarrhythmic drugs provided any reduction in stroke rate when compared with placebo. ${ }^{9}$ A post hoc analysis of the AFFIRM data revealed that although maintenance of sinus rhythm was associated with better survival, this benefit was neutralized by increased mortality resulting from antiarrhythmic drug use. ${ }^{10}$ A criticism that is often raised in regard to AFFIRM and AF-CHF is the fact that both trials were under-represented in regard to percentage of geriatric subjects enrolled. One would expect that precisely this age population is most at risk for developing arrhythmia. Other antiarrhythmic drugs, such as dofetilide and dronedarone, have been primarily studied in elderly patients. This could potentially give them a leading edge over the older drugs when prescribing for the geriatric population. The commonly prescribed dose of dronedarone (400 $\mathrm{mg}$ bid) has been extensively studied in a patient population with atrial fibrillation and of mean age 72 years, in which more than $80 \%$ of subjects were older than 65 years. ${ }^{11}$

\section{Physiological changes in the geriatric population}

The myocardium experiences electrical and structural changes, such as increased fibrosis resulting in decreased ventricular compliance, as a direct consequence of aging. This loss of ventricular compliance translates into increased diastolic filling pressures, which in turn facilitate the development of atrial fibrillation. ${ }^{12}$ The increased stiffness of the ventricular myocardium inhibits inflow and causes not only elevated filling pressures but also a progressive increase in atrial diameter. Electrophysiologically, in older age, the action potential duration and the effective refractory period of both atrial and ventricular tissue become longer, whereas conduction at the atrioventricular junction slows. ${ }^{13}$

In elderly people, the arrhythmia source is also changed by scarring resulting from myocardial infarction, ischemia, and increased stretch. Other possible etiologies include ventricular, valvular, and systemic disorders (viral infections, systemic amyloidosis, ankylosing spondylitis) resulting in collagen, amyloid, and fibrosis formation, which ultimately contribute to generation of arrhythmia.

\section{Pharmacokinetics, pharmacodynamics, and pharmacogenetics in the elderly}

Limited data are available from clinical studies regarding the pharmacokinetics and pharmacodynamics of antiarrhythmic drugs in elderly subjects. Age-related changes in pharmacokinetics and pharmacodynamics make the elderly more prone to the development of adverse drug reactions, especially when incremental comorbidities are taken into account. Consequently, many elderly patients suffer from polypharmacy, making drug interactions more likely. The altered pharmacokinetics of aging change the effects of absorption, bioavailability, distribution, and clearance of drugs.

\section{Absorption}

The geriatric population has increased gastric $\mathrm{pH}$, secondary to atrophic changes in the gastric mucosa, with diminished acid secretion capacity and reduced gastrointestinal blood flow. Thus, elevated gastric $\mathrm{pH}$ is common in the elderly and can affect the ionization and solubility of certain drugs. Reduced acidity can also provoke more rapid emptying of the stomach contents into the duodenum. An agerelated decline in gastrointestinal motility has also been described. ${ }^{14}$

\section{Bioavailability}

Due to a decreased first-pass extraction, the bioavailability of some drugs with a high rate of first-pass metabolism may be increased.

\section{Distribution}

Changes in body composition can affect drug disposition. There is an age-related increase in body fat, decrease in lean body mass, and decrease in total body water. These alterations can result in an increased volume of distribution of lipid-soluble drugs and a diminished volume of distribution for water-soluble drugs. In the elderly, serum albumin levels may decline, with the total plasma protein content being unaffected. A reduction in albumin levels can result in an increase in the free drug fraction, which is the pharmacodynamically active part of the medication. $\alpha 1$ acid glycoprotein binds mostly to basic lipophilic drugs and this tends to increase with age. The binding of drugs to $\alpha 1$ acid glycoprotein also increases during an acute illness, such as myocardial infarction, and is a factor that needs to be taken into consideration by the prescribing physician. 


\section{Hepatic metabolism}

Several changes in liver structure and function have been noted in the elderly. There is an absolute decrease in the size of the liver and a reduction in hepatic blood flow. The most frequently seen changes involve the microsomal mixedfunction oxidative system (phase I, oxidation and reduction), with little or no change in conjugative processes (phase II, conjugation). Nevertheless, sometimes liver microsomal mono-oxygenase levels remain unaltered despite increasing age, perhaps due to interindividual variability. Another area of interest is hepatic enzyme induction in the elderly. There is still controversy as to whether drug induction is an important phenomenon in the geriatric population. Cytochrome P450 (CYP) 2D6 inhibitors, such as quinidine, amiodarone, dronedarone, paroxetine, and propafenone, have been shown to diminish the metabolism of metoprolol, resulting in higher than expected plasma concentrations. Strong CYP3A4 inhibitors, such as ketoconazole, are associated with a marked increase in maximum dronedarone concentration and are thus contraindicated, and inducers of CYP3A4 will conversely decrease dronedarone exposure. Some antiepileptic drugs, like carbamazepine and phenytoin, as well as certain antibiotics, such as rifampicin, induce metabolism by increasing the induction of CYP3A4. This leads to subtherapeutic and ineffective concentrations of quinidine, disopyramide, and dronedarone. Dronedarone is a substrate for P-glycoprotein and will lead to an increase in concentration of P-glycoprotein substrates, such as digoxin. ${ }^{15}$

\section{Excretion}

Glomerular filtration rate gradually declines with age. At the same time, there is a decrease in renal plasma flow, causing the filtration fraction to rise significantly with age. Loss of tubular function and diminished reabsorptive capacity are also observed with aging. Owing to reduced muscle mass, evaluation of renal function with serum creatinine alone can be misleading in elderly subjects. ${ }^{16} \mathrm{~A}$ more precise way to estimate glomerular filtration rate is by estimation of the endogenous creatinine clearance using the Cockcroft and Gault equation, which is a very useful tool for dose adjustment of renally excreted drugs:

$e C_{C r}=\frac{(140-\text { Age }) \times \text { Mass (in kilograms }) \times[0.85 \text { if Female }]}{72 \times \text { Serum Creatinine }(\text { in } \mathrm{mg} / \mathrm{dL})}$

When serum creatinine is measured in $\mu \mathrm{mol} / \mathrm{L}$ :

$$
e C_{C r}=\frac{(140-\text { Age }) \times \text { Mass (in kilograms }) \times \text { Constant }}{\text { Serum Creatinine }(\text { in } \mu \mathrm{mol} / \mathrm{L})}
$$

While the concomitant use of diuretics, nephrosclerosis due to hypertension, general anesthesia, and presence of congestive heart failure commonly encountered in elderly patients can lead to further renal compromise, age remains an independent risk factor for deterioration of renal function. In summary, all these factors that impair renal drug clearance can lead to drug accumulation and toxic effects.

\section{Pharmacogenomics}

The role of pharmacogenetics in daily clinical practice is increasing as practical guidelines regarding gene-drug interactions become more available. ${ }^{17}$ The aim of pharmacogenetics is to establish connections between pharmacology and genetics, in particular connections between pharmacological phenotypes and genotypes, to predict individual responses to drug treatments. The term pharmacogenetics is not recent, and was introduced in the early 1960s by Vogel, ${ }^{18}$ based on earlier studies of person-to-person variability in response to drug treatment. ${ }^{19}$ Since that time, several studies have shown that the CYP enzyme system is responsible for the metabolism of more than $80 \%$ of commercially available drugs. ${ }^{20}$ Therefore, interindividual differences seen in the response to treatment, as well as in the appearance of drug side effects, are caused by variations in CYP encoding genes. Due to polypharmacy in the elderly population, adverse drug reactions are an important cause of morbidity and mortality.

Most currently prescribed drugs derive from natural plant metabolites, and are metabolized by enzymes encoded by CYP genes, which show a high rate of polymorphism. ${ }^{21}$ Regarding the evolution of drug-metabolizing enzymes, the occurrence of a large number of duplications in CYP genes was documented about 300-400 million years ago.

The CYP enzymes encoded by CYP genes are part of a highly polymorphic gene superfamily, thus the high rate of polymorphism displayed by these genes leads to marked variability in the activity of the encoded enzymes. For this reason, CYP enzymes play an important role in drug metabolism, determining drug bioavailability, and therefore the clinical response to pharmacological treatments.

Clinicians should be aware of the implications when prescribing drugs to the elderly, and consider the current knowledge of pharmacogenetics in their clinical practice. Some investigators advocate that differences in the genetics of CYP will lead to personalized drug treatments, with a special emphasis on the geriatric population. 


\section{Factors to consider in antiarrhythmic treatment for the elderly}

Given that aging is associated with an increased risk of adverse effects, selection of antiarrhythmic drugs in the elderly is basically determined by factors such as the treatment target, assumed patient compliance, possible interaction with other medications the patient is concomitantly taking for comorbidities (eg, hypertension, congestive heart failure), and renal and liver function. Therefore, prescription of antiarrhythmic drugs in the elderly often involves dosage modification. As a general rule, a 50\% reduction in the starting dose of an antiarrhythmic drug compared with younger patients would seem to be a wise approach. However, this does not apply to dronedarone (which has been specifically studied in an elderly population) or dofetilide.

\section{Amiodarone: properties and side effect profile}

Amiodarone is generally considered the gold standard in antiarrhythmic therapy and has the best efficacy in terms of maintaining normal sinus rhythm in patients with atrial fibrillation. ${ }^{22}$ Nevertheless, its use is often limited by its side effect profile. Potentially life-threatening proarrhythmic adverse drug reactions have been described with amiodarone, such as torsades de pointes, but their incidence seems to be very low. ${ }^{23,24}$

The most common cardiovascular side effects include bradycardia, atrioventricular conduction abnormalities, and QT prolongation. First degree heart block and bradyarrhythmia have been reported..$^{25}$

However, in the setting of heart failure, amiodarone has been recommended because of its low proarrhythmic potential and lack of a significant negative inotropic effect in congestive heart failure. ${ }^{26}$ The presence of left ventricular systolic dysfunction does not significantly affect the outcome, indicating the utility of amiodarone for controlling atrial fibrillation in patients with congestive heart failure.

Dronedarone, a newer drug, has no proarrhythmic potential, and almost no incidence of torsades de pointes, with only one case in ATHENA (A placebo-controlled, double-blind, parallel arm Trial to assess the efficacy of dronedarone $400 \mathrm{mg}$ twice daily for the prevention of cardiovascular Hospitalisation or death from any cause in patiENts with Atrial fibrillation/ atrial flutter). Data suggest that concomitant administration of dronedarone and digoxine results in an increase in maximal concentrations of digoxine. ${ }^{27}$ Thus, amiodarone has a relatively safe cardiac adverse drug reaction profile..$^{28}$
Pulmonary toxicity is a very common noncardiac side effect. ${ }^{29}$ The prevalence is estimated to be about $5 \% .{ }^{30}$ Although amiodarone-induced pulmonary toxicity can occur at any time after treatment is initiated, those considered at greatest risk are individuals who have received a daily dose of $400 \mathrm{mg}$ for more than 2 months, or a lower dose, commonly $200 \mathrm{mg}$ daily, for more than 2 years. In the earliest descriptions of amiodarone, the reported incidence of amiodaroneinduced pulmonary toxicity was $5 \%-15 \%$ when patients were taking $400 \mathrm{mg}$ or more per day. ${ }^{31}$ Subsequently, lower doses were used in an attempt to avoid toxicity. As emphasized by Polkey et al, there is probably no safe dose of this medication, and this complication continues to be reported. ${ }^{32}$

Hypersensitivity pneumonitis can appear early in the course of therapy. Interstitial pneumonitis is a more common but insidious pulmonary reaction characterized by cough, low-grade fever, and dyspnea that occurs after months or years of therapy.

The overall incidence of amiodarone-induced thyroid dysfunction is about $15 \%$. The incidence of amiodaroneinduced hypothyroidism ranges from $1 \%-31 \%$, and it is more frequent in iodine-sufficient areas. The presence of antibodies against thyroid peroxidase, an enzyme in thyroid hormone metabolism, is related to an increased risk of developing hypothyroidism during treatment with amiodarone (seven-fold in men and 13-fold in women). Therefore, it may be advisable to measure thyroid peroxidase antibodies at the start of amiodarone treatment. The reported incidence of amiodarone-induced thyrotoxicosis is in the range of $1 \%-23 \%$. Amiodarone-induced thyrotoxicosis develops in $3 \%$ of amiodarone-treated patients in North America, and is classified as type 1 or type 2 . Type 1 disease occurs in patients with underlying thyroid pathology, such as autonomous nodular goiter or Graves' disease. Type 2 disease is a result of amiodarone causing a subacute thyroiditis with release of preformed thyroid hormones into the circulation. ${ }^{33}$ Amiodarone also inhibits type $15^{\prime}$-deiodinase activity in peripheral tissue. Amiodarone and its metabolites demonstrate direct toxicity in cultured thyroid cells exposed to media with concentrations above those normally found in patients. ${ }^{34}$ The effects of amiodarone on the thyroid can be seen as early as a few weeks after starting treatment and/or up to several months after its discontinuation. ${ }^{35}$

Because thyroid dysfunction is relatively common in amiodarone therapy, all patients should have free thyroxine and thyroid-stimulating hormone levels measured before starting therapy, at 3-4-month intervals during treatment, and for at least 1 year after amiodarone is discontinued. 
Optic neuritis or neuropathy, which causes patients to experience decreased or blurred vision, may progress in exceptionally rare cases to permanent blindness. Nearly all patients have microdeposits of amiodarone in the cornea, but this is rarely a reason to discontinue the drug. ${ }^{36}$ This is a dose-independent phenomenon and is reversible after cessation of the drug. Halo vision, especially in the dark, can be disturbing, and lowering of the dose is generally effective in the long term. Optic neuritis is very rare, and usually starts within 12 months of initiation of treatment, at a median of 4 months. If optic neuritis develops, amiodarone must be discontinued.

Hepatotoxicity is a relatively uncommon side effect of amiodarone. ${ }^{37}$ Although asymptomatic elevation of aminotransferases is reported in up to $25 \%$ of patients, symptomatic hepatic dysfunction occurs in less than $1 \%$ of patients on chronic amiodarone therapy. ${ }^{38}$ Risk factors for developing hepatotoxicity are not defined. It may be difficult to prove the pathogenic role of amiodarone in a patient developing liver disease while on therapy with this drug. ${ }^{39}$ It is also not known whether patients with underlying asymptomatic chronic liver disease are predisposed to amiodarone-induced hepatotoxicity. Clinically, amiodarone hepatotoxicity occurs in two settings, ie, with use of rapid intravenous infusion of amiodarone in the intensive care setting to control arrhythmias and with long-term oral use. The former usually presents as severe acute hepatitis with a massive rise in transaminases, occasionally leading to acute liver failure. Most cases recover after the infusion is discontinued. However, fatal instances of progressive liver failure have occurred. Although controversial, it is believed that this acute reaction is an effect of polysorbate 80 , which is used to solubilize the intravenous preparation of amiodarone, rather than the drug per se. ${ }^{40}$ This is consistent with the fact that oral amiodarone has been successfully used in patients who have developed acute hepatitis after intravenous use of amiodarone. ${ }^{41}$ However, chronic amiodarone toxicity usually presents as mild asymptomatic elevation of liver enzymes. It is usually alanine aminotransferase and aspartate aminotransferase that are elevated to 2-10 times the upper limit of normal. ${ }^{42}$ Other liver chemistries (alkaline phosphatase, gamma-glutamyl transferase, bilirubin, and lactate dehydrogenase have not been shown to vary significantly in patients on chronic amiodarone therapy. Aminotransferase elevation is usually reversible after dose reduction or drug discontinuation, although it may take anywhere between 3 weeks and 9 months for liver biochemistry to normalize. It is not known whether continued therapy in patients with mildly elevated liver enzymes leads to progressive and symptomatic hepatic dysfunction. Symptomatic liver disease develops in only $1 \%-3 \%$ of patients. With prolonged treatment, amiodarone hepatotoxicity may insidiously progress to cirrhosis and present with signs and symptoms of decompensated liver failure, portal hypertension, and laboratory evidence of hypoprothrombinemia and hypoalbuminemia, although this is rare..$^{43}$ A mean cumulative dose of $380 \mathrm{~g}$ was found in patients with hepatotoxicity leading to cirrhosis. Cirrhosis, although uncommon, has been well documented. ${ }^{44}$

Several types of dermatological reactions have been reported, including allergic rash, photosensitivity, and blue-gray skin discoloration. ${ }^{45}$ Neurological side effects are uncommon, and include tremor, ataxia, peripheral neuropathy, malaise or fatigue, sleep disturbances, dizziness, and headaches.

In summary, amiodarone is a safe and efficacious antiarrhythmic agent when lower doses are used in patients who are closely monitored and subjected to careful follow-up. However, it is imperative that physicians are aware of these potentially severe drug-induced complications.

\section{Dronedarone, a new antiarrhythmic drug}

Dronedarone (Sanofi-Aventis, Bridgewater, NJ) is the first antiarrhythmic drug used in the treatment of atrial fibrillation and atrial flutter that has been shown to reduce cardiovascular hospitalizations ${ }^{46}$ Dronedarone is also a potent coronary vasodilator in the hearts of rabbits, guinea pigs, and rats via nitric oxide synthase-mediated and guanylyl cyclasemediated effects. ${ }^{47}$

After oral administration, $70 \%-94 \%$ of dronedarone is absorbed in the postprandial state, with absorption increasing by up to three-fold when it is taken with food (especially high-fat meals). Debutyldronedarone, formed primarily by CYP3A4, is a major circulating metabolite with similar or even higher plasma exposure than dronedarone..$^{48}$ Debutyldronedarone has been demonstrated to be pharmacodynamically active but exhibits a potency that is $10 \%-33 \%$ that of the parent drug. The majority of the absorbed dronedarone is eliminated via first-pass metabolism, with only $15 \%$ bioavailability. ${ }^{49}$ Steady-state plasma concentrations of $84-167 \mathrm{ng} / \mathrm{mL}$ are reached in 1 week by administration of dronedarone $400 \mathrm{mg}$ twice daily. ${ }^{50}$ The primary metabolic clearance of dronedarone is driven by hepatic CYP3A4-dependent mechanisms, with a terminal half-life of approximately 24 hours. ${ }^{51}$ 
Dronedarone also interacts with commonly prescribed drugs, such as metoprolol and simvastatin. ${ }^{52}$ For example, dronedarone can increase serum simvastatin levels by 2-4-fold, and promote statin-induced myopathy. The interaction between dronedarone and metoprolol depends on CYP2D6 inhibition and results in increased bioavailability of metoprolol. Dronedarone also has interactions with other drugs using the CYP system, so should not be administered with potent CYP3A4 inhibitors, including antifungals, certain oral macrolide antibiotics, and protease inhibitors, because CYP3A4 inhibition may increase plasma levels of dronedarone and cause unwanted adverse effects. However, dronedarone can be coadministered with moderate CYP3A4 inhibitors, such as verapamil and diltiazem, but with some caution, eg, by using lower drug doses.

Although dronedarone has no effect on glomerular filtration rate, slightly increased creatinine levels are reported in patients treated with dronedarone. These can be explained by partial inhibition of tubular transport of creatinine..$^{53}$ Some of the available data concerning the molecular and electrophysiological mechanisms of action and preclinical efficacy of dronedarone are discussed below.

Dronedarone was approved by the US Food and Drug Administration in March 2009 to reduce the risk of cardiovascular hospitalization in patients with paroxysmal or persistent atrial fibrillation or atrial flutter. Approval was justified due to the positive results of the ATHENA trial showing significant reductions in all-cause mortality and cardiovascular hospitalization with the use of dronedarone. ${ }^{47}$ Dronedarone was developed to accommodate the need for a potent antiarrhythmic drug without the side effects of amiodarone. Its electrophysiological properties are similar to amiodarone, but there are several structural differences. ${ }^{54}$

Dronedarone is primarily a class III antiarrhythmic drug but it has properties from all four classes according to the Vaughan-Williams classification of antiarrhythmic drugs. It also has antiadrenergic properties and inhibits multiple transmembrane potassium currents, including the delayed rectifier current (both the rapid and slow components [IKr and IKs, respectively]), the ultrarapid delayed rectifier current (IKur), the inward rectifier current (IK1), the transient outward current (ITo), and the sodium and L-type calcium currents. Dronedarone has a methylsulfonamide group, making it less lipophilic than amiodarone and thus having a much shorter half-life, with reduced tissue accumulation. ${ }^{55}$ The European Medicines Agency Committee for Medicinal Products for Human Use has approved dronedarone for use in clinically stable adult patients with a history of or current nonpermanent atrial fibrillation, to prevent recurrence of atrial fibrillation, or to lower the ventricular rate. ${ }^{56}$ The available data show that no dose reductions are required for dronedarone.

In rabbit and guinea pig heart models, both amiodarone and dronedarone prolong the ventricular and atrial refractory periods, suppress the automaticity of the sinoatrial node due to prolongation of the action potential, and decrease the maximum slope of the action potential upstroke, which reflects blocking of the fast sodium channel activity of the myocardium. Dronedarone slows the sinus rate and causes a dose-dependent lengthening of the corrected QT interval, and, with escalating doses, causes prolongation of the PR interval. In spite of these similarities, the antagonistic effects of the two drugs are not equivalent. In vitro data show that dronedarone has a stronger inhibitory effect on the peak sodium current and on the acetylcholine-activated potassium current than amiodarone..$^{57}$

\section{Clinical trials with dronedarone}

All clinical studies with dronedarone have been multinational, multicenter, double-blind, placebo-controlled, and of parallel design with comparable demographics, except for the DIONYSOS (Efficacy and safety of dronedarone versus amiodarone for the maintenance of sinus rhythm in patients with atrial fibrillation) trial which was actively controlled.$^{58}$ There have been seven trials undertaken which were designed to help establish the efficacy, dosage, and rate control achieved with dronedarone. These clinical trials are categorized by their primary intention, ie, according to whether they are studies of rhythm control, rate control, mortality/morbidity, or comparative efficacy.

\section{Initial dose-ranging trial}

DAFNE (the Dronedarone Atrial FibrillatioN study after Electrical cardioversion) was a prospective, double-blind, randomized, placebo-controlled Phase II study performed in patients with persistent atrial fibrillation, in whom cardioversion and drug therapy were warranted. The study was designed to establish the optimal therapeutic dose of dronedarone for rhythm control of atrial fibrillation. A total of 270 patients, aged $21-85$ years, with persistent atrial fibrillation, were randomized to three different doses of dronedarone (400 mg, $600 \mathrm{mg}$, or $800 \mathrm{mg}$ twice daily) or placebo, and were then followed for 6 months. Patients with unstable angina, recent myocardial infarction, New York Heart Association (NYHA) class III-IV congestive heart failure or left ventricular ejection fraction less than $35 \%$ were excluded. If they failed to convert to sinus rhythm within 
5-7 days of starting dronedarone therapy, the patients were electrically cardioverted. The primary endpoint was the time to first recurrence of atrial fibrillation during the 6 months of follow-up. Secondary endpoints included spontaneous conversion of atrial fibrillation, heart rate in atrial fibrillation, and occurrence of adverse events.

Dronedarone $400 \mathrm{mg}$ twice daily was found to prolong the period to first recurrence of the arrhythmia significantly, and was better tolerated with fewer side effects. In DAFNE, dronedarone was not associated with thyroid, pulmonary, neurological, ocular, or pulmonary toxicity. Dronedarone treatment led to dose-dependent prolongation of the QT interval, but no cases of torsades de pointes were reported. Patients treated with the highest doses had more gastrointestinal toxicity leading to drug discontinuation. After 6 months, the percentage of patients on dronedarone $400 \mathrm{mg}$ twice daily and back in atrial fibrillation was $65 \%$ vs $90 \%$ for those on placebo. The median time to reversion back to atrial fibrillation was 60 days vs 5 days for placebo $(P=0.001)$. In summary, the DAFNE study demonstrated the safety and moderate efficacy of dronedarone $400 \mathrm{mg}$ twice daily for the management of persistent atrial fibrillation. ${ }^{59}$ Limitations of DAFNE included the investigators' inability to explain fully the lack of a dose-related response to dronedarone and the relatively high recurrence rate of atrial fibrillation.

\section{Rate control of atrial fibrillation}

Dronedarone has also been studied as a rate-controlling agent for atrial fibrillation. The ERATO trial (Efficacy and safety of dRonedArone for The control of ventricular rate during atrial fibrillation) enrolled 174 subjects (older than 21 years, predominantly elderly males $>65$ years) with permanent atrial fibrillation of more than 6 months' duration and had a 6-month follow-up period. The patients were randomized to receive $800 \mathrm{mg}$ of dronedarone daily or placebo. ${ }^{27}$

At the time of first recurrence of atrial fibrillation, dronedarone appeared to slow the ventricular response in a dose-dependent fashion. Patients receiving dronedarone 800,1200 , or $1600 \mathrm{mg}$ had their ventricular rate reduced by $13.2,19.2$, and 17.8 beats per minute on average, respectively, compared with those on placebo $(P=0.0001)$. The results after 4 months of follow-up showed that the rate-controlling effect of dronedarone was sustained over time. The efficacy of dronedarone therapy also appeared to be additive to the effects of conventional background rate-controlling therapy, including calcium antagonists, beta-blockers, and digoxin. There were no untoward interactions between dronedarone and other rate-controlling agents or anticoagulants, except for a $41 \%$ increase in serum digoxin concentrations.

In conclusion, dronedarone is an effective rate controlling agent, both at rest and during exercise, without negative effects on exercise tolerance. In patients who suffer recurrence of atrial fibrillation, dronedarone may decrease symptoms and consequently lower hospital admissions and emergency room visits.

\section{Maintaining normal sinus rhythm}

EURIDIS (the EURopean trial In atrial fibrillation or flutter patients receiving Dronedarone for the maintenance of Sinus rhythm) and ADONIS (the American-Australian-African trial with DrOnedaroNe in atrial fibrillation or flutter patients for the maintenance of Sinus rhythm) were sister trials that evaluated dronedarone for the maintenance of sinus rhythm in 828 patients who received dronedarone and 409 patients who received placebo, each patient group aged over 21 years, with a follow-up duration of 12 months. ${ }^{60}$ Time to recurrence of atrial fibrillation, which was the primary endpoint of the trial, was significantly longer in patients treated with dronedarone. Furthermore, heart rate at first recurrence of atrial fibrillation was lowered significantly with dronedarone. A post hoc analysis revealed a $27 \%$ reduction of relative risk of hospitalization and death with dronedarone treatment. The rates of cardiac and extracardiac adverse events in these trials were comparable with those of the placebo arm. There was a reported incidence of serum creatinine elevation of $2.4 \%$ in patients in the dronedarone group.

\section{Effect on morbidity/mortality}

The AFFIRM trial, which included 4060 patients, showed that rhythm control strategies do not translate into a survival benefit. AFFIRM found no significant difference in mortality or stroke risk between the treatment strategies of rate or rhythm control. ${ }^{61}$ A post hoc analysis of the AFFIRM data revealed that although maintenance of sinus rhythm was associated with better survival, this benefit was neutralized by increased mortality from antiarrhythmic drug use. ${ }^{10}$

The ATHENA study evaluated 4628 high-risk patients with paroxysmal or persistent atrial fibrillation/atrial flutter. High risk was defined as patients older than 75 years with at least one of the following: hypertension, diabetes, prior cerebrovascular accident or systemic embolism, left ventricular ejection fraction $<40 \%$ or left atrial diameter $>50 \mathrm{~mm} .^{58,62}$ Patient characteristics and drug treatment were similar in both arms, although it must be noted that the ATHENA trial was not designed as a head-to-head comparison between 
dronedarone and amiodarone. The primary endpoint was defined as first hospitalization for cardiovascular reasons or mortality from any cause.

Thirty-two percent of patients in the dronedarone arm and $39 \%$ in the placebo arm reached the primary endpoint. Dronedarone reduced the first cardiovascular hospitalization by $26 \%$ when compared with placebo $(P<0.001)$. The reduction in need for repeated hospitalization for cardiovascular events was due to fewer hospital admissions for treatment of atrial fibrillation or acute coronary syndromes. All-cause mortality was similar in both study arms. Discontinuation of the study drug was similar in both groups, indicating good tolerability. Dronedarone proved to be effective and safe in reducing cardiovascular outcomes (cardiovascular hospitalization or death) in patients with atrial fibrillation or flutter. A post hoc analysis of the AFFIRM data revealed that although maintenance of sinus rhythm was associated with better survival, this benefit was neutralized by increased mortality from antiarrhythmic drug use. ${ }^{10}$ Baseline risk factors for stroke were well balanced between the two groups, and the percentage of patients receiving oral anticoagulants with therapeutic INR was also similar. The baseline risk of stroke was assessed using the CHADS2 score, which is calculated by assigning one point each for the presence of congestive heart failure, hypertension, age 75 years or older, and diabetes mellitus, and two points for history of stroke or transient ischemic attack. ${ }^{62}$ Patients included in the study had an intermediate to high risk for stroke and other cardiovascular events, with a mean CHADS2 score of 2 in both groups. Patients with a CHADS2 score $\geq 2$ had a significantly greater effect of dronedarone than those with a CHADS2 score $\leq 1$ ( $P=0.03$ for interaction). Dronedarone reduced the risk of stroke by $34 \%$ from $1.8 \%$ per year to $1.2 \%$ per year (hazards ratio [HR]: $0.66, P=0.027$ ). Consequently, this analysis showed a reduction of stroke with dronedarone in high-risk patients. ${ }^{62}$

\section{Patients with heart failure}

The ANDROMEDA trial (ANti-arrhythmic trial with DROnedarone in Moderate-to-severe congestive heart failure Evaluating morbidity DecreAse) was designed to evaluate the effect of dronedarone on morbidity in patients with unstable NYHA class II-IV heart failure, regardless of their arrhythmia history. ${ }^{63}$ After inclusion of 627 patients (310 in an $800 \mathrm{mg}$ daily dronedarone group and 317 in a placebo group), the trial was prematurely terminated for safety reasons, at the recommendation of the Data and Safety Monitoring Board, in accordance with the board's predefined rules for study termination. During a median follow-up of 2 months, 25 patients in the dronedarone group $(8.1 \%)$ and 12 patients in the placebo group (3.8\%) died (HR in the dronedarone group 2.13; 95\% confidence interval [CI]: 1.07-4.25; $P=0.03)$. The excess mortality was predominantly related to worsening of heart failure, with 10 deaths in the dronedarone group and two in the placebo group. The primary endpoint did not differ significantly between the two groups; there were $53(17.1 \%)$ events in the dronedarone group and $40(12.6 \%)$ events in the placebo group (HR: 1.38; 95\% CI: 0.92-2.09; $P=0.12$ ). More increases in creatinine levels were reported as serious adverse events in the dronedarone group than in the placebo group. The risk of death and hospitalization was higher in patients with the most severe left ventricular systolic dysfunction. The study was stopped prematurely due to increased mortality in the dronedarone group, primarily due to worsening heart failure. There is now a black box warning in the package insert against the use of this drug in patients with NYHA class IV heart failure or class II-III heart failure with recent decompensation requiring hospitalization or referral to a heart failure specialist. As a direct result of the ANDROMEDA trial, dronedarone is contraindicated in patients with NYHA class III and IV or in patients with recent exacerbation of heart failure.

The ATHENA trial included patients with NYHA class IIIII heart failure and a left ventricular ejection fraction $<40 \%$ at baseline (114 on placebo and 95 on dronedarone). Unlike prior studies, this trial had a composite primary endpoint of all-cause mortality and cardiovascular hospitalization. ${ }^{64}$ A primary outcome event occurred in 59/114 placebo patients compared with $42 / 95$ patients receiving dronedarone. Twenty of 114 placebo patients and twelve of 95 dronedarone patients died during the study. Fifty-four placebo patients and 42 dronedarone patients were hospitalized for an intermittent episode of NYHA class IV heart failure. There was a trend toward lower overall mortality with dronedarone treatment, and, importantly, there was a statistically significant reduction in death due to cardiac arrhythmia. A post hoc analysis demonstrated that dronedarone was associated with a significant reduction in the adjusted risk of stroke compared with placebo. ${ }^{62}$ This benefit was preserved in patients who were already receiving antithrombotic therapy. ${ }^{65}$ Due to the risk of torsades de pointes associated with sotalol and dofetilide, physicians have typically waited 1 month or more to starting these drugs after stopping amiodarone. In the ATHENA trial, patients had to stop amiodarone at least 1 month prior to enrollment in the trial. The earlier EURIDIS and ADONIS trials allowed patients to be enrolled immediately after 
discontinuation of amiodarone. Physicians are currently using their clinical judgment in deciding on this question, but no one is advocating this switch in patients whose arrhythmia is well controlled if they are not experiencing any adverse effects on amiodarone.

\section{Dronedarone versus amiodarone}

When initial results were discussed with the European Agency for the Evaluation of Medicinal Products, it was requested that a comparison be made with amiodarone. This was done using DIONYSOS, a multicenter, randomized, double-blind, parallel-arm study comparing dronedarone $400 \mathrm{mg}$ twice daily with amiodarone $600 \mathrm{mg}$ daily for 28 days and $200 \mathrm{mg}$ daily thereafter, for the maintenance of sinus rhythm in patients with atrial fibrillation. In total, 504 patients of mean age $64.0 \pm 10.7$ years were randomly allocated to dronedarone $(n=249)$ or amiodarone $(n=255)$, and electrical cardioversion was performed if necessary. Patients could be included if they had documented atrial fibrillation for more than 72 hours and required cardioversion and antiarrhythmic therapy. Patients with NYHA class III-IV congestive heart failure were excluded. The primary endpoint was a composite time to first recurrence of atrial fibrillation or premature discontinuation of the study drug because of intolerance or lack of efficacy.

At a mean follow-up of 7 months, fewer amiodaronetreated patients reached the primary endpoint compared with those treated with dronedarone $(55.3 \%$ vs $73.9 \%, P<0.001)$, indicating that amiodarone showed more sustained efficacy than dronedarone. Patients on amiodarone tended to experience more premature drug discontinuation (34 vs 26) than patients on dronedarone. At 12 months, more patients given dronedarone had recurrence of atrial fibrillation or prematurely stopped taking the study drug $(75.1 \%$ vs $58.8 \%$, HR: 1.589, 95\% CI: 1.275-1.980; log rank $P$ value $<0.0001)$. Amiodarone was found to be more effective than dronedarone in maintaining sinus rhythm.

The main safety endpoint in DIONYSOS was time to first occurrence of thyroid, hepatic, pulmonary, neurological, skin, ocular, or specific gastrointestinal events, or premature discontinuation of the study drug because of any adverse effects. This happened in $39.3 \%$ of patients taking dronedarone and $44.5 \%$ of those taking amiodarone after 12 months (HR: 0.80, CI: 0.60-1.07; $\log \operatorname{rank} P$ value $=0.13$ ). Patients treated with dronedarone had a significantly higher prevalence of adverse gastrointestinal events and a significantly lower incidence of thyroid (two vs 15) and adverse neurological events (three vs 17).
Intolerance of dronedarone was mainly secondary to gastrointestinal side effects (diarrhea, vomiting, and nausea), and was responsible for discontinuation of the drug in only $3.2 \%$ of patients in clinical trials, while thyroid and neurological events (tremor and sleep disorder) were mostly responsible for discontinuation of amiodarone. ${ }^{66}$ Fewer patients in the dronedarone group had a QTc interval $>500$ milliseconds ( $10.9 \%$ vs $20.5 \%$, respectively). No cases of torsades de points were reported in either study arm. The incidence of recurrence of atrial fibrillation in the dronedarone arm was higher than in the amiodarone arm, whereas adverse events leading to premature drug discontinuation tended to be less frequent in the dronedarone arm. These factors, along with fewer hospitalizations and decreased stroke risk seen in the ATHENA trial, may result in a decreased cost of treatment in dronedarone patients. However, no cost-efficacy analyses have been done to confirm this theory.

The superiority of amiodarone in preventing recurrence of atrial fibrillation is consistent with an indirect meta-analysis based on placebo-controlled trials of amiodarone and placebocontrolled trials of dronedarone. ${ }^{67}$ They included direct randomized data obtained from the DIONYSOS trial, together with other controlled studies that randomized patients with atrial fibrillation to amiodarone, dronedarone, or placebo. ${ }^{67}$ The results of this comparison showed that treatment with amiodarone was associated with a greater likelihood of remaining in sinus rhythm (odds ratio [OR]: for recurrent atrial fibrillation 0.49 , 95\% CI: $0.37-0.63 ; P<0.001)$ in comparison with dronedarone. On the other hand, the authors also observed a trend toward greater all-cause mortality (OR: 1.61, 95\% CI: $0.97-2.68 ; P=0.066)$ and a greater number of adverse events requiring drug discontinuation with amiodarone versus dronedarone (OR: 1.81, 95\% CI: 1.33-2.46; $P<0.001) .{ }^{67}$ In conclusion, DIONYSOS clearly confirms that dronedarone is less effective than amiodarone in maintaining sinus rhythm and in preventing recurrence of atrial fibrillation in patients with a history of atrial fibrillation. Dronedarone can be considered as an alternative therapy to amiodarone and tried prior to amiodarone, especially in younger patients.

\section{Safety of dronedarone}

Because CAST (the Cardiac Arrhythmia Suppression Trial) showed that antiarrhythmic therapy with class IC drugs increases mortality in patients after myocardial infarction, there has been a justified focus on the safety of antiarrhythmic drugs. Currently, amiodarone is the only antiarrhythmic drug that is used in high-risk patients, even though it is not approved for this indication by the Food and Drug 
Table I Adverse drug reactions more frequent than placebo

\begin{tabular}{lll}
\hline & $\begin{array}{l}\text { Placebo } \\
(\mathbf{n}=\mathbf{2 8 7 5})\end{array}$ & $\begin{array}{l}\text { Dronedarone } \\
\mathbf{4 0 0} \text { mg twice daily } \\
(\mathbf{n}=\mathbf{3 2 8 2})\end{array}$ \\
\hline $\begin{array}{ll}\text { Gastrointestinal } \\
\quad \text { Diarrhea }\end{array}$ & \\
$\quad$ Nausea & $6 \%$ & $9 \%$ \\
$\quad$ Abdominal pain & $3 \%$ & $5 \%$ \\
$\quad$ Vomiting & $3 \%$ & $4 \%$ \\
$\quad$ Dyspeptic signs and symptoms & $1 \%$ & $2 \%$ \\
General & $1 \%$ & $2 \%$ \\
$\quad$ Asthenic conditions & $5 \%$ & $7 \%$ \\
Cardiac & & \\
$\quad$ Bradycardia & $1 \%$ & $3 \%$ \\
Skin and subcutaneous tissue & & \\
$\quad$ Including rashes (generalized, & $3 \%$ & $5 \%$ \\
$\quad$ macular, maculopapular, & & \\
$\quad$ erythematous), pruritus, eczema, & & \\
$\quad$ dermatitis, dermatitis allergic & & \\
\hline
\end{tabular}

Data from Touboul P, Brugada J, Capucci A, Crijns HJ, Edvardsson N, Hohnloser SH. Dronedarone for prevention of atrial fibrillation: a dose-ranging study. Eur Heart J. 2003;24(16):148|-1487.

Administration. It often causes prolongation of the QT interval and in some cases torsades de pointes. The extracardiac safety problems reported with amiodarone, in particular thyroid, eye, skin, nervous system, liver, respiratory, thoracic, and mediastinal disorders, were reported in comparable frequencies with dronedarone and placebo. This argues in favor of a better extracardiac safety profile for dronedarone than amiodarone. The DIONYSOS study showed that premature study drug discontinuation following an adverse event tended to occur less frequently in the dronedarone group than in the amiodarone group. Dronedarone prolongs the QT interval, but the risk of torsades de pointes is low. The DYONISOS trial did not report any cases of torsades de pointes, and only one case was reported in the ATHENA trial.

Dronedarone should not be used in conjunction with other drugs that prolong the QT interval. While periodic electrocardiograms are recommended to monitor patients for a prolonged QT interval and bradycardia, no chest X-rays or laboratory tests to monitor thyroid and hepatic function are required. Since there is no significant interaction with warfarin, more frequent monitoring of coagulation is not needed. Patients should take dronedarone with food to increase absorption, and should avoid grapefruit juice, which can increase serum levels of this drug. Dronedarone has not been studied in pregnant women. However, the drug has been found to be teratogenic in animal studies.

The DYONISOS study suggested a better safety profile for dronedarone, chiefly due to fewer thyroid, neurological, skin, and ocular events. The most common side effects of dronedarone are gastrointestinal, including diarrhea and nausea, which are more frequent than with amiodarone or with placebo (Table 1). Photosensitivity reactions and dysgeusia have also been reported, with an incidence of less than $1 \%$ in patients treated with dronedarone. Laboratory data and electrocardiographic parameters reported with dronedarone tablets $400 \mathrm{mg}$ twice daily are shown in Table 2.

Dronedarone increases serum creatinine by $10 \%-15 \%$, but without adverse effects on glomerular filtration rate or renal plasma flow in patients and healthy individuals. This is due to partial inhibition of tubular organic cation transporters, and this is also seen during treatment with amiodarone.

With respect to vitamin $\mathrm{K}$ antagonists, drug interactions were less frequent with dronedarone than with amiodarone, with fewer hemorrhagic events. Notwithstanding, some drug interactions may be relevant. Plasma levels of dronedarone may be increased several-fold in patients taking a strong inhibitor of CYP3A4, like ketoconazole, itraconazole, voriconazole, telithromycin, clarithromycin, ciclosporin, and ritonavir, and these drugs are contraindicated in such situations. On the other hand, during treatment with dronedarone, potent CYP3A4 inducers, such as phenobarbital, carbamazepine, phenytoin, or St John's wort, are not recommended because they decrease dronedarone exposure up to five-fold. Dronedarone $400 \mathrm{mg}$ twice daily increased simvastatin and simvastatin acid exposure 2-4-fold, respectively, and increased exposures of lovastatin, atorvastatin, and pravastatin within the same range as simvastatin. Although interaction with some statins (CYP3A4 substrates) could hypothetically lead to increased risk of statin-related adverse events, especially myopathy, data from the ATHENA trial and the integrated analyses did not confirm this assumption. Dronedarone may increase the plasma levels of some beta-blockers (metoprolol, propranolol) and calcium channel blockers (verapamil, diltiazem), and dose adjustments of beta-blocker treatment may be needed after introduction of dronedarone. In the ERATO

Table 2 Laboratory data and electrocardiographic parameters not necessarily reported as adverse events

\begin{tabular}{lll}
\hline & Placebo & $\begin{array}{l}\text { Dronedarone } \\
\mathbf{4 0 0} \mathbf{~ m g} \text { twice daily }\end{array}$ \\
\hline Serum creatinine increased $\geq 10 \%$ & $(\mathrm{n}=\mathbf{2 8 7 5})$ & $(\mathrm{n}=3282)$ \\
5 days after treatment initiation & $21 \%$ & $51 \%$ \\
QTc Bazett prolonged & $(\mathrm{n}=2237)$ & $(\mathrm{n}=270 \mathrm{I})$ \\
$\begin{array}{l}(>450 \text { milliseconds in males } \\
\text { and }>470 \text { milliseconds in females })\end{array}$ & $19 \%$ & $28 \%$ \\
\hline
\end{tabular}

Data from Touboul P, Brugada J, Capucci A, Crijns HJ, Edvardsson N, Hohnloser SH. Dronedarone for prevention of atrial fibrillation: a dose-ranging study. Eur Heart J. 2003;24(|6): : $48 \mid-1487$. 


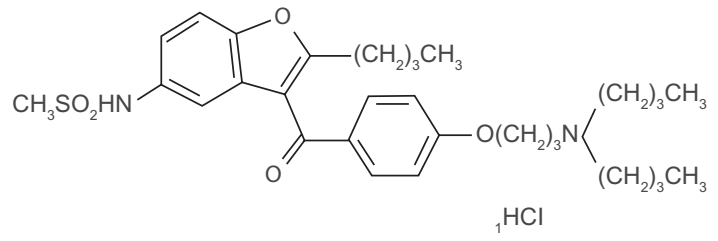

Figure I Chemical structure of dronedarone.

Notes: Chemical structure of the noniodinated benzofuran derivative of amiodarone, dronedarone. N-[2-butyl-3-[4-[3-(dibutylamino)propoxy]benzoyl]-I-benzofuran-5-yl]methanesulfonamide.

trial, the mean increase in digoxin levels in the dronedarone arm compared with placebo was $41.4 \%$, but the number of patients with digoxin levels outside the normal range in the two groups did not differ significantly.

Although no hepatic toxicity was detected during Phase III trials of dronedarone, post-marketing surveillance has recently identified two cases of severe hepatic failure requiring transplantation amongst approximately 180,000 patients exposed worldwide. No definite causal relationship between dronedarone and liver injury has been established, but close monitoring of liver function tests is recommended.

\section{Conclusion}

Dronedarone, like amiodarone, has effects on multiple cardiac ion channels and receptors. This agent can be considered as a first-line therapy for atrial fibrillation in patients with structural heart disease who have an ejection fraction $>35 \%$ and no recent decompensated heart failure. Dronedarone has been proven to maintain sinus rhythm and to control the ventricular rate during episodes of atrial fibrillation in several clinical trials. In ATHENA, dronedarone reduced cardiovascular hospitalizations and mortality in high-risk patients with atrial fibrillation.

Dronedarone has a well-described side-effect profile, the principal adverse effect being diarrhea, which may necessitate drug discontinuation. Dronedarone causes dose-dependent prolongation of the QTc interval, but torsades de pointes is rare. The drug increases serum creatinine by inhibition of tubular secretion. This effect is not associated with reduced renal function and is reversible, but needs to be considered, particularly in patients receiving other drugs like angiotensinconverting enzyme inhibitors that also increase serum creatinine. The safety of dronedarone in patients with advanced heart failure is a concern. Patients with severe systolic heart failure and hemodynamic instability should not receive dronedarone. Theoretically, dronedarone use should result in lower health care cost for atrial fibrillation patients due to its favorable safety profile, lowering of stroke risk, and reduction in cardiovascular hospitalizations. The ultimate role for dronedarone is yet to be defined. Little evidence exists as to whether it will succeed when other drugs have failed.

\section{Disclosure}

The authors report no conflicts of interest in this work.

\section{References}

1. Riley AB, Manning WJ. Atrial fibrillation: an epidemic in the elderly. Expert Rev Cardiovasc Ther. 2011;9:1081-1090.

2. Stewart S, Hart CL, Hole DJ, McMurray JJ. Population prevalence, incidence, and predictors of atrial fibrillation in the Renfrew/Paisley study. Heart. 2001;86:516-521.

3. Go AS, Hylek EM, Phillips KA, et al. Prevalence of diagnosed atrial fibrillation in adults: national implications for rhythm management and stroke prevention: the AnTicoagulation and Risk Factors in Atrial Fibrillation (ATRIA) Study. JAMA. 2001;285:2370-2375.

4. Cairns JA, Connolly S, McMurtry S, Stephenson M, Talajic M; CCS Atrial Fibrillation Guidelines Committee. Canadian Cardiovascular Society atrial fibrillation guidelines 2010: prevention of stroke and systemic thromboembolism in atrial fibrillation and flutter. Can J Cardiol. 2011;27:74-90.

5. Gattellari M, Goumas C, Aitken R, Worthington JM. Outcomes for patients with ischaemic stroke and atrial fibrillation: The PRISM Study (A Program of Research Informing Stroke Management). Cerebrovasc Dis. 2011;32:370-382.

6. Wolf PA, Abbott RD, Kannel WB. Atrial fibrillation as an independent risk factor for stroke: the Framingham study. Stroke. 1991;22:983-988.

7. Roger VL, Go AS, Lloyd-Jones DM, et al. American Heart Association Statistics Committee and Stroke Statistics Subcommittee. Heart disease and stroke statistics - 2011 update: a report from the American Heart Association. Circulation. 2011;12:e18-e209.

8. Piccini JP, Wallace TW, Patel MR, Becker RC. Stroke prevention in atrial fibrillation. Cardiovasc Drugs Ther. 2011;25(6):561-570.

9. Le Heuzey JY, Marijon E, Otmani A, et al. Treatment of atrial fibrillation in heart failure. Therapie. 2009;64:115-119. French.

10. Corley SD, Epstein AE, DiMarco JP, et al; AFFIRM Investigators. Relationships between sinus rhythm, treatment, and survival in the Atrial Fibrillation Follow-Up Investigation of Rhythm Management (AFFIRM) study. Circulation. 2004;109:1509-1513.

11. Hohnloser SH, Crijns HJ, van EM, et al. Effect of dronedarone on cardiovascular events in atrial fibrillation. $N$ Engl J Med. 2009;360: 668-678.

12. Deneer VH, van Hemel NM. Is antiarrhythmic treatment in the elderly different? A review of the specific changes. Drugs Aging. 2011;28: 617-633.

13. Huang CC, Sandroni P, Sletten DM, Weigand SD, Low PA. Effect of age on adrenergic and vagal baroreflex sensitivity in normal subjects. Muscle Nerve. 2007;36:637-642.

14. Bender A. Effect of age on intestinal absorption: implications for drug absorption in the elderly. J Am Geriatr Soc. 1968;16:1331-1339.

15. Dorian P. Clinical pharmacology of dronedarone: implications for the therapy of atrial fibrillation. J Cardiovasc Pharmacol Ther. 2010; 15(4 Suppl):15S-18S

16. Rowe JW, Andres R, Tobin JD, Norris AH, Shock NW. The effect of age on creatinine clearance in men: a cross-sectional and longitudinal study. J Gerontol. 1976;31:155-163.

17. Swen JJ, Wilting I, de Goede AL, et al. Pharmacogenetics: from bench to byte. Clin Pharmacol Ther. 2008;83:781-787.

18. Vogel F. [Modern problems of human genetics]. Inn Med Kinderheilkd. 1959;12:52-125. German.

19. Alving AS, Carson PE, Flanagan CL, Ickes CE. Enzymatic deficiency in primaquine sensitive erythrocytes. Science. 1956;124:484-485.

20. Seripa D, Pilotto A, Panza F, Matera MG, Pilotto A. Pharmacogenetics of cytochrome P450 (CYP) in the elderly. Ageing Res Rev. 2010;9:457-474 
21. Ingelman-Sundberg M. Pharmacogenomic biomarkers for prediction of severe adverse drug reactions. N Engl J Med. 2008;358:637-639.

22. Kawabata M, Hirao K, Hachiya H, et al. Role of oral amiodarone in patients with atrial fibrillation and congestive heart failure. J Cardiol. 2011;58:108-115.

23. van Erven L, Schalij MJ. Amiodarone: an effective antiarrhythmic drug with unusual side effects. Heart. 2010;96:1593-1600.

24. Connolly SJ. Evidence-based analysis of amiodarone efficacy and safety. Circulation. 1999;100:2025-2034.

25. Vorperian VR, Havighurst TC, Miller S, January CT. Adverse effects of low dose amiodarone: a meta-analysis. J Am Coll Cardiol. 1997;30:791-798.

26. Camm AJ, Kirchhof P, Lip GY, et al. European Heart Rhythm Association, European Association for Cardio-Thoracic Surgery. Guidelines for the management of atrial fibrillation: the task force for the management of atrial fibrillation of the European Society of Cardiology (ESC). Europace. 2010;12:1360-1420.

27. Davy JM, Herold M, Hoglund C, et al; ERATO Study Investigators. Dronedarone for the control of ventricular rate in permanent atrial fibrillation: the Efficacy and safety of dRonedArone for The cOntrol of ventricular rate during atrial fibrillation (ERATO) study. Am Heart J. 2008;156:527. e1-e9.

28. Bongard V, Marc D, Philippe V, Jean-Louis M, Maryse LM. Incidence rate of adverse drug reactions during long-term follow-up of patients newly treated with amiodarone. Am J Ther. 2006;13:315-319.

29. Wolkove N, Baltzan M. Amiodarone pulmonary toxicity. Can Respir J. 2009; 16:43-48.

30. Schwaiblmair M, Berghaus T, Haeckel T, Wagner T, von Scheidt W. Amiodarone-induced pulmonary toxicity: an under-recognized and severe adverse effect? Clin Res Cardiol. 2010;99:693-700.

31. Jessurun GA, Boersma WG, Crijns HJ. Amiodarone-induced pulmonary toxicity. Predisposing factors, clinical symptoms and treatment. Drug Saf. 1998;18:339-344.

32. Polkey MI, Wilson PO, Rees PJ. Amiodarone pneumonitis: no safe dose. Respir Med. 1995;89:233-235.

33. Tsang W, Houlden RL. Amiodarone-induced thyrotoxicosis: a review. Can J Cardiol. 2009;25:421-424.

34. Chiovato L, Martino E, Tonacchera M, et al. Studies on the in vitro cytotoxic effects of amiodarone. Endocrinology. 1994;134:2277-2282.

35. Cardenas GA, Cabral JM, Leslie CA. Amiodarone-induced thyrotoxicosis: Diagnostic and therapeutic strategies. Cleve Clin J Med. 2003;70:624-631.

36. Mantyjarvi M, Tuppurainen K, Ikaheimo K. Ocular side effects of amiodarone. Surv Ophthalmol. 1998;42:360-366.

37. Raja K, Thung SN, Fiel MI, Chang C. Drug-induced steatohepatitis leading to cirrhosis: long-term toxicity of amiodarone use. Semin Liver Dis. 2009;29:423-428.

38. Babatin M, Lee SS, Pollak PT. Amiodarone hepatotoxicity. Curr Vasc Pharmacol. 2008;6:228-236.

39. Llanos L, Moreu R, Peiró AM, et al. Causality assessment of liver injury after chronic oral amiodarone intake. Pharmacoepidemiol Drug Saf. 2009; 18:291-300.

40. Giannattasio F, Salvio A, Varriale M, et al. Three cases of severe acute hepatitis after parenteral administration of amiodarone: the active ingredient is not the only agent responsible for hepatotoxicity. Ann Ital Med Int. 2002; 17:180-184. Italian.

41. James PR, Hardman SM. Acute hepatitis complicating parenteral amiodarone does not preclude subsequent oral therapy. Heart. 1997;77:583-584.

42. Kum LC, Chan WW, Hui HH, et al. Prevalence of amiodarone-related hepatotoxicity in 720 Chinese patients with or without baseline liver dysfunction. Clin Cardiol. 2006;29:295-299.

43. Stravitz RT, Sanyal AJ. Drug-induced steatohepatitis. Clin Liver Dis. 2003; 7:435-451.

44. Puli SR, Fraley MA, Puli V, Kuperman AB, Alpert MA. Hepatic cirrhosis caused by low-dose oral amiodarone therapy. Am J Med Sci. 2005;330:257-261.
45. Hilleman D, Miller MA, Parker R, Doering P, Pieper JA. Optimal management of amiodarone therapy: efficacy and side effects. Pharmacotherapy. 1998;18(6 Pt 2):138S-145S

46. Torp-Pedersen C, Crijns HJ, Gaudin C, Page RL, Connolly SJ, Hohnloser SH; ATHENA Investigators. Impact of dronedarone on hospitalization burden in patients with atrial fibrillation: results from the ATHENA study. Europace. 2011;13:1118-1126.

47. Guiraudou P, Pucheu SC, Gayraud R, et al. Involvement of nitric oxide in amiodarone- and dronedarone-induced coronary vasodilation in guinea pig heart. Eur J Pharmacol. 2004;496:119-127.

48. Xie C, Yang S, Zhong D, Dai X, Chen X. Simultaneous determination of dronedarone and its active metabolite debutyldronedarone in human plasma by liquid chromatography-tandem mass spectrometry: Application to a pharmacokinetic study. J Chromatogr B Analyt Technol Biomed Life Sci. 2011;879:3071-3075.

49. Pamukcu B, Lip GY. Dronedarone as a new treatment option for atrial fibrillation patients: pharmacokinetics, pharmacodynamics and clinical practice. Expert Opin Pharmacother. 2011;12:131-140.

50. US Food and Drug Administration. Highlights of prescribing information. Available from: http://www.accessdata.fda.gov/drugsatfda_docs/ label/2009/022425lbl.pdf. Accessed October 14, 2011.

51. European Medicines Agency. Multaq; Annex I Summary of product characteristics. Available from: http:/www.ema.europa.eu/docs/en_ GB/document_library/EPAR_-_Product_Information/human/001043/ WC500044534.pdf. Accessed October 14, 2011.

52. Damy T, Pousset F, Caplain H. Pharmacokinetic and pharmacodynamic interactions between metoprolol and dronedarone in extensive and poor CYP2D6 metabolizers healthy subjects. Fundam Clin Pharmacol. 2004;18:113-123.

53. Tschuppert Y, Buclin T, Rothuizen LE. Effect of dronedarone on renal function in healthy subjects. Br J Clin Pharmacol. 2007;64:785-791.

54. Zareba KM. Dronedarone: a new antiarrhythmic agent. Drugs Today (Barc). 2006;42:75-86.

55. Schweizer PA, Becker R, Katus HA, Thomas D. Dronedarone: current evidence for its safety and efficacy in the management of atrial fibrillation. Drug Des Devel Ther. 2011;5:27-39.

56. Rosei EA, Salvetti M. Dronedarone: an emerging therapy for atrial fibrillation. Ther Adv Cardiovasc Dis. 2010;4:155-164.

57. Patel C, Yan GX, Kowey PR. Dronedarone. Circulation. 2009;120: 636-644.

58. Le Heuzey JY, De Ferrari GM, Radzik D, Santini M, Zhu J, Davy JM. A short-term, randomized, double-blind, parallel-group study to evaluate the efficacy and safety of dronedarone versus amiodarone in patients with persistent atrial fibrillation: the DIONYSOS study. J Cardiovasc Electrophysiol. 2010;21:597-605.

59. Touboul P, Brugada J, Capucci A, Crijns HJ, Edvardsson N, Hohnloser SH. Dronedarone for prevention of atrial fibrillation: a doseranging study. Eur Heart J. 2003;24:1481-1487.

60. Singh BN, Connolly SJ, Crijns HJ, et al. Dronedarone for maintenance of sinus rhythm in atrial fibrillation or flutter. $N$ Engl J Med. 2007;357:987-999.

61. Freudenberger RS, Wilson AC, Kostis JB; AFFIRM Investigators and Committees. Comparison of rate versus rhythm control for atrial fibrillation in patients with left ventricular dysfunction. Am J Cardiol. 2007; 100:247-252.

62. Connolly SJ, Crijns HJ, Torp-Pedersen C, et al; ATHENA Investigators. Analysis of stroke in ATHENA: a placebo-controlled, double-blind, parallel-arm trial to assess the efficacy of dronedarone $400 \mathrm{mg}$ BID for the prevention of cardiovascular hospitalization or death from any cause in patients with atrial fibrillation/atrial flutter. Circulation. 2009; 120:1174-1180.

63. Kober L, Torp-Pedersen C, McMurray JJ, et al. Increased mortality after dronedarone therapy for severe heart failure. $N$ Engl J Med. 2008;358:678-687.

64. Naccarelli GV, Wolbrette DL, Khan M. Old and new antiarrhythmic drugs for converting and maintaining sinus rhythm in atrial fibrillation: comparative efficacy and results of trials. Am J Cardiol. 2003;91:15D-26D. 
65. Dagres N, Sommer P, Anastasiou-Nana M, Hindricks G. Treating arrhythmias: an expert opinion. Expert Opin Pharmacother. 2011;12: 1359-1367.

66. Cook GE, Sasich LD, Sukkari SR. Atrial fibrillation. DIONYSOS study comparing dronedarone with amiodarone. BMJ. 2010;340:c285.
67. Piccini JP, Hasselblad V, Peterson ED, Washam JB, Califf RM, Kong DF. Comparative efficacy of dronedarone and amiodarone for the maintenance of sinus rhythm in patients with atrial fibrillation. $\mathrm{J} \mathrm{Am} \mathrm{Coll}$ Cardiol. 2009;54:1089-1095.

\section{Publish your work in this journal}

Clinical Interventions in Aging is an international, peer-reviewed journal focusing on evidence-based reports on the value or lack thereof of treatments intended to prevent or delay the onset of maladaptive correlates of aging in human beings. This journal is indexed on PubMed Central, MedLine, the American Chemical Society's 'Chemical Abstracts

\section{Dovepress}

Service' (CAS), Scopus and the Elsevier Bibliographic databases. The manuscript management system is completely online and includes a very quick and fair peer-review system, which is all easy to use. Visit $\mathrm{http}: / /$ www.dovepress.com/testimonials.php to read real quotes from published authors.

Submit your manuscript here: http://www.dovepress.com/clinical-interventions-in-aging-journal 